\title{
Insulating behavior with spin and charge order in the ionic Hubbard model
}

\author{
Krzysztof Byczuk, ${ }^{1}$ Michael Sekania, ${ }^{2}$ Walter Hofstetter, ${ }^{3}$ and Arno P. Kampf ${ }^{2}$ \\ ${ }^{1}$ Institute of Theoretical Physics, University of Warsaw, ul. Hoża 69, PL-00-681 Warszawa, Poland \\ ${ }^{2}$ Theoretical Physics III, Center for Electronic Correlations and Magnetism, \\ Institute of Physics, University of Augsburg, D-86135 Augsburg, Germany \\ ${ }^{3}$ Institut für Theoretische Physik, Johann Wolfgang Goethe-Universität, D-60438 Frankfurt/Main, Germany
}

(Dated: May 26, 2022)

\begin{abstract}
Paramagnetic solutions of the ionic Hubbard model at half-filling in dimensions $D>2$ indicate that the band and the Mott insulator phases are separated by a metallic phase. We present zero-temperature dynamical mean-field theory solutions, which include antiferromagnetic long-range order, and show that the one-particle spectral functions always possess an energy gap and therefore the system is insulating for all interaction strengths. The staggered charge density modulation coexists with antiferromagnetic long-range order of Néel type.
\end{abstract}

PACS numbers: 71.27.+a, 71.30.+h, 71.10.Fd

A bipartite lattice system of non-interacting electrons with one particle per site is a perfect gapless metal. Applying an external alternating potential with a periodicity of twice the lattice constant $a$ doubles the unit cell, thereby reduces the Brillouin zone (BZ), and opens a gap at the BZ boundary. Such a system is a band insulator with a charge density modulation with wavelength $2 a$; this system is also referred to as an ionic insulator: 1 On the other hand, switching on a local repulsive interaction between the electrons with opposite spins leads either to a paramagnetic Mott-Hubbard insulator with a correlation induced energy gap or to the spontaneous development of antiferromagnetic (AF) long range order. In the latter case it is the presence of $\mathrm{AF}$ order which doubles the unit cell and opens a gap at the BZ boundary for weak interactions and thereby creates a Slater insulator. In the strong interaction limit the electrons are localized with antiferromagnetically aligned spins forming a Mott-Heisenberg insulator. Experimental and theoretical investigations of metal-insulator transitions and transitions between different insulators continue as a challenge for condensed matter physics.

The ionic Hubbard model ${ }^{2,3}$ incorporates both interactions and an external alternating potential and is therefore well suited to study transitions between metallic or different insulating phases. This model was originally used to study the neutral-ionic transition in organic charge transfer salts ${ }^{3}$ or ferroelectric transitions in perovskite materials ${ }^{4}$ But the understanding of possible phase transitions in the ionic Hubbard model may prove important for other strongly correlated electron systems as well such as, for example, FeSi $\stackrel{5}{\frac{5}{n}}$ The physics of the ionic Hubbard model may even find a realization in optical lattices, if two laser beams of commensurate wavelengths are superposed with properly tuned amplitudes $\underline{\underline{6}}$

Extensive literature records exact, approximate, as well as numerical results for the ionic Hubbard model in one dimension ${ }^{7.8 .9}$ It is agreed that at half-filling and in the interaction dominated regime the system is a paramagnetic Mott insulator whereas in the alternating potential dominated regime the system is an ionic band insulator. By now there is an emergent consensus that these two types of insulators are separated by yet another insulating phase with a non-zero bond-order parameter, which is the expectation value for a staggered component of the kinetic energy.

More recently the ionic Hubbard model was also investigated in higher dimensions within single-site or cluster dynamical mean-field theory (DMFT) $)^{10,11,12,13}$ or by determinant quantum Monte-Carlo simulations. $\frac{14}{\underline{\underline{14}}}$ Using DMFT with iterated perturbation theory as the tool for solving the DMFT equations, Garg et al. determined the ground-state phase diagram of the ionic Hubbard model at half-filling for a semicircular density of states $\stackrel{11}{=}$ With the restriction to paramagnetic solutions an intermediate metallic phase was found separating the Mott and the band insulator. Within the same computational framework Craco et al. identified a coexisting phase between two insulators as well as discontinuous metal-insulator transitions $\stackrel{12}{12}$ The discontinuous transitions were confirmed in the two dimensional (2D) system by Kancharla et al. $\stackrel{13}{\frac{13}{2}}$ who used cluster DMFT combined with exact diagonalization and interpreted their data in favor of an intermediate bond ordered phase.

While the DMFT work was restricted to paramagnetic solutions, finite temperature quantum Monte Carlo in $2 \mathrm{D}$ simulations also probed AF correlations $\underline{\underline{14}}$ The presence of the intermediate metallic phase between the band and the Mott insulator was confirmed. However, since in the $2 \mathrm{D}$ system at finite temperature long range antiferromagnetism is prohibited,,$\frac{15}{w}$ the question how the possible presence of the antiferromagnetic long-range order in higher dimensions or in $2 D$ at zero temperature changes the phase diagram has remained open.

Here we apply DMFT to the ionic Hubbard model at zero temperature allowing for spontaneous AF long-range order. We find that the ground state is always insulating, with a gap in the one-particle spectral function. There is a direct transition between the band insulator and the AF Mott insulator. Beyond a critical interaction strength the charge- and the spin-density modulations coexist. In this region the insulator has $\mathrm{AF}$ character. 
The ionic Hubbard model on a bipartite lattice is defined by the following Hamiltonian

$$
H=-t \sum_{\langle i j\rangle \sigma} a_{i \sigma}^{\dagger} a_{j \sigma}+U \sum_{i} n_{i \uparrow} n_{i \downarrow}+\sum_{i \sigma} \Delta_{i} n_{i \sigma},
$$

where $\Delta_{i}= \pm \Delta / 2$ for $i \in A, B$ sublattices, respectively. The first term describes the kinetic energy of the electrons with spin $\sigma= \pm 1 / 2$ for the hopping between nearest-neighbor lattice sites $i$ and $j$ with amplitude $t$, the Hubbard interaction leads to an energy increase $U \geq 0$ for the double occupancy of a site, and the last term contains a staggered potential with an energy difference $\Delta \geq 0$ between the $A$ and $B$ sublattices. The operators $a_{i \sigma}$ and $a_{i \sigma}^{\dagger}$ obey standard fermionic anticommutation relations and $n_{i \sigma}=a_{i \sigma}^{\dagger} a_{i \sigma}$.

The Hamiltonian (11) is solved within DMFT at halffilling, i.e. for the chemical potential $\mu=U / 2$, by a mapping to two non-equivalent impurity problems with the ionic energies $\pm \Delta / 2,16,17,18,19$ The impurity sites are coupled to two particle baths. Explicitly, we solve separately the two different single-impurity Anderson models

$$
\begin{aligned}
H_{\text {SIAM }}^{\alpha} & =\left(\epsilon_{\alpha}-\mu\right) n_{\alpha \sigma}+\sum_{k} V_{k \alpha \sigma}\left(a_{\alpha \sigma}^{\dagger} c_{k \alpha \sigma}+h . c .\right) \\
& +U n_{\alpha \uparrow} n_{\alpha \downarrow}+\sum_{k} \epsilon_{k \alpha \sigma} c_{k \alpha \sigma}^{\dagger} c_{k \alpha \sigma},
\end{aligned}
$$

where the hybridization matrix elements $V_{k \alpha \sigma}$ and the kinetic energies of the bath electrons $\epsilon_{k \alpha \sigma}$ are obtained self-consistently by additional DMFT equations. $\frac{19}{19}$ Here we explicitly keep the spin and the site $(\alpha=A, B)$ dependences in order to allow selectively for spin and charge order. The one-particle impurity energy is $\epsilon_{\alpha}= \pm \Delta / 2$ for $\alpha=A$ or $B$, respectively. The single-impurity Anderson Hamiltonians are solved at zero temperature by the numerical renormalization group (NRG) method ${ }^{20}$ This method allows to obtain the spectral functions at and near the Fermi energy with high precision and can therefore accurately distinguish between metallic and insulating phases ${ }^{21}$

The local (impurity) Green functions obtained from (2) are expressed via the hybridization function $\eta_{\alpha \sigma}(\omega)$ and the self-energies $\Sigma_{\alpha \sigma}(\omega)$ as

$$
G_{\alpha \sigma}(\omega)=\frac{1}{\omega-\left(\epsilon_{\alpha}-\mu\right)-\eta_{\alpha \sigma}(\omega)-\Sigma_{\alpha \sigma}(\omega)} .
$$

The hybridization functions describe the resonant broadening of the impurity energy levels due to the coupling to the particle baths and are given by

$$
\eta_{\alpha \sigma}(\omega)=\sum_{k} \frac{V_{k \alpha \sigma}}{\omega-\epsilon_{k \alpha \sigma}} .
$$

The self-energies capture the interaction induced correlation effects on the impurity sites.

Within DMFT the hybridization functions are subject to self-consistency conditions, which involve the density

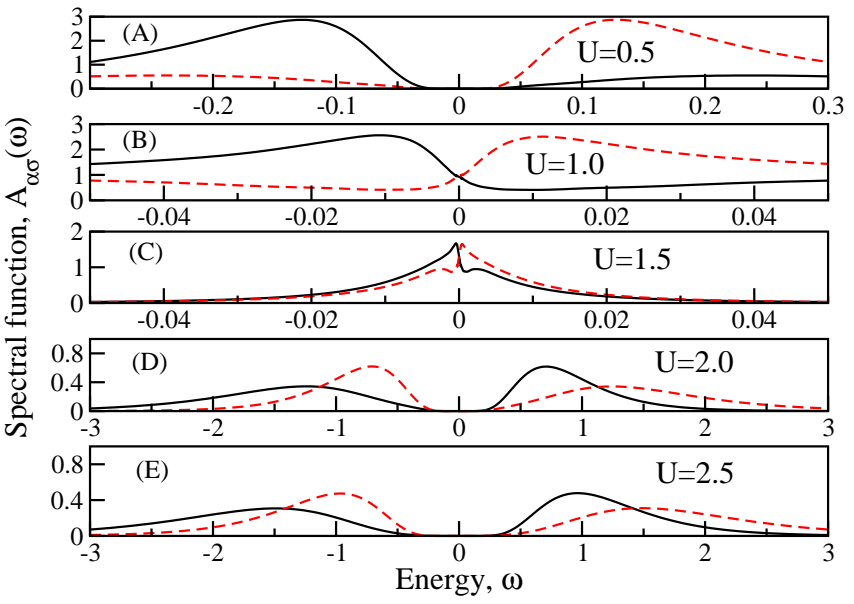

FIG. 1: (color online) Spectral functions for the ionic Hubbard model at $\Delta=0.5$ and different interactions $U$ in the paramagnetic limit. Solid and dashed lines correspond to the A and B sublattices. From top to bottom: (A) band insulator; (B), (C) correlated metal; (D), (F) correlated Mott insulators. Note the different scales on the axis in particular horizontal ones.

of states (DOS) for a given lattice structure ${ }^{17,18,19}$ In the following we adopt the semicircular DOS corresponding to the Bethe lattice, $19,22,23$ The hybridization functions are then simply related to the local Green functions (3) through

$$
\eta_{\alpha \sigma}(\omega)=\frac{W^{2}}{16} G_{\bar{\alpha} \bar{\sigma}}(\omega),
$$

where $\bar{\alpha}=B, A$ if $\alpha=A, B$ and $\bar{\sigma}=-\sigma$, respectively. $W=1$ is the bandwidth, which sets the energy unit. We emphasize that the use of the semicircular DOS has merely technical reasons because this choice simplifies the DMFT equations. The obtained results remain qualitatively similar for any particle-hole symmetric DOS, which represents a bipartite lattice in dimensions $D>2.22,23$

From the self-consistent DMFT solution of the ionic Hubbard model we determine (i) the local one-particle spectral function $A_{\alpha \sigma}(\omega)=-\operatorname{Im} G_{\alpha \sigma}(\omega) / \pi$, (ii) the $\mathrm{AF}$ order parameter (staggered magnetization) $m_{\mathrm{AF}}=$ $\left\langle n_{A \uparrow}-n_{A \downarrow}\right\rangle=-\left\langle n_{B \uparrow}-n_{B \downarrow}\right\rangle$, and (iii) the charge density wave amplitude (ionicity) $m_{\mathrm{CDW}}=\left\langle n_{A \uparrow}+n_{A \downarrow}-\right.$ $\left.n_{B \uparrow}-n_{B \downarrow}\right\rangle$, where $\langle\cdots\rangle$ denotes ground-state expectation values. A metal is distinguished from an insulator by a finite spectral function at the Fermi level, i.e. $A(0)=\sum_{\alpha \sigma} A_{\alpha \sigma}(0)>0$.

Without allowing for long-range $\mathrm{AF}$ order in the selfconsistent DMFT solution the results of iterated perturbation theory suggested that the band and the Mott insulator are separated by a metallic phase $\stackrel{11,12}{=}$ Here, we confirm this conclusion by using NRG for solving the DMFT equations. In Fig. 1 we show spectral functions in the paramagnetic regime at fixed $\Delta=0.5$ starting from a band insulator at small interactions (cf. top panel with $U=0.5)$. By increasing $U$ we find a continuous tran- 


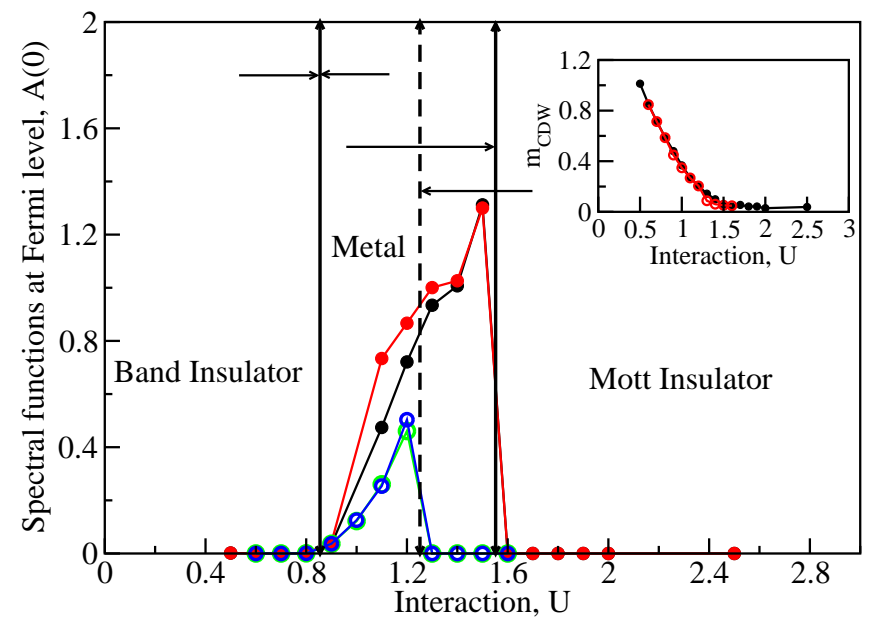

FIG. 2: (color online) Spectral functions at the Fermi energy versus $U$ for the ionic Hubbard model at $\Delta=0.5$ in the paramagnetic limit. Solid data points (black and red) are obtained from DMFT iterations which start from an initial metallic input whereas open points (blue and green) are obtained from an insulating input. The band insulator to metal transition is continuous, but the metal to Mott insulator transition is hysteretic. Inset: charge density wave amplitude $m_{\mathrm{CDW}}$ vs. $U$.

sition to a metallic solution with finite spectral weight at the Fermi energy (for $U=1$ and 1.5 in Fig. 1B and C). This metallic phase coexists with long-range charge order, i.e. $m_{\mathrm{CDW}}>0$. Larger interaction strengths homogenize the system and the charge density wave amplitude continuously decreases (cf. the results for $U=1.5$ in Fig. 1C and the inset in Fig. 21). A further increase of $U$ leads to a Mott-Hubbard type metal-insulator transition with hysteretic behavior $\stackrel{21.24}{ }$ In the examples shown in Fig. 1 for $U=2$ and 2.5 we find Mott insulators with correlation induced spectral gaps. In the Mott insulator the charge density wave is very small but remains finite.

The two transitions are separated in the phase diagram and imply the existence of two critical interaction strengths 11,12 The transition from the metal to the Mott insulator resembles the one, which is found within DMFT applied to the paramagnetic Hubbard model in the absence of a staggered potential. ${ }^{21,24}$ At zero temperature this transition is continuous, though hysteretic behavior in the iterative solution is encountered; it occurs at $U_{c} \approx 1.45$ for a semicircular DOS.

We note that although $m_{\mathrm{CDW}}$ becomes vanishingly small for large $U$ (see the inset in Fig. 2) it is expected to remain finite for all interaction strengths as long as $\Delta>0$. This expectation relies on the general argument that the symmetry of the ground state cannot be higher than the symmetry of the Hamiltonian itself.

It is understood, however, that the paramagnetic solution of the Hubbard model at half-filling is not the generic case. Antiferromagnetism is likely to occur unless it is prohibited e.g. by strong frustration effects. In the next step we therefore discuss the ionic Hubbard model at

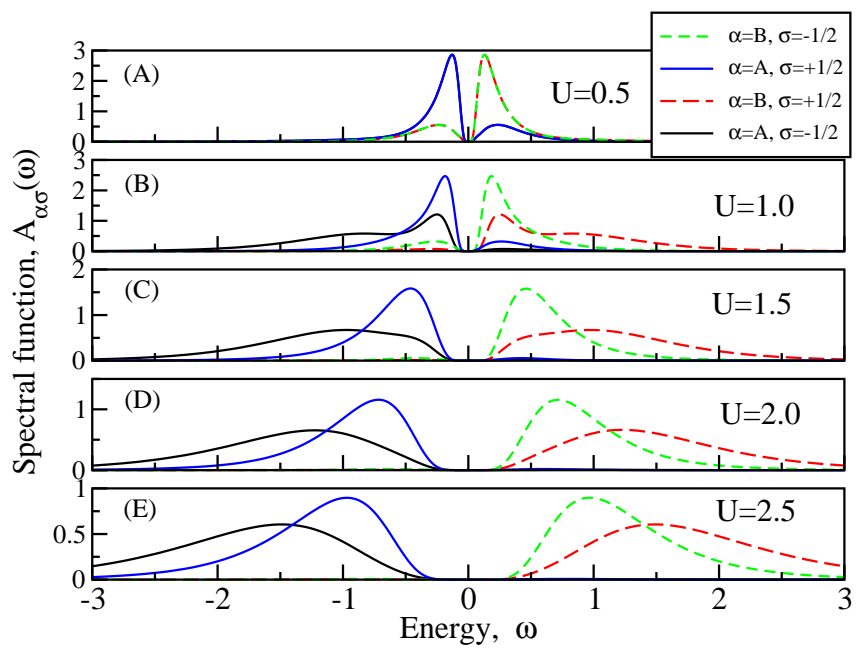

FIG. 3: (color online) Spectral functions of the ionic Hubbard model for $\Delta=0.5$ and different interactions $U$ allowing for AF order. Solid and dashed lines correspond to different sublattices, the color code distinguishes the spins $\sigma= \pm 1 / 2$, as indicated in the inset. (A) Paramagnetic band insulator with $m_{\mathrm{CDW}}>0$ and $m_{\mathrm{AF}}=0$. (B) - (E) Antiferromagnetic ionic insulator with $m_{\mathrm{CDW}}>0$ and $m_{\mathrm{AF}}>0$. Note different scales on vertical axis. In all cases the spectra are gapped.

half-filling on a bipartite lattice allowing for long-range antiferromagnetism.

Both, staggered charge order, induced by the alternating potential $\Delta$, and spontaneous staggered AF order may give rise to a gapped spectrum and insulating behavior of the lattice system at half-filling. If both staggered orders develop simultaneously, the above mentioned metallic phase in between the ionic band and the Mott insulator may be insulating due to an AF induced energy gap. Indeed, as the examples in Fig. 3 illustrate, the spectral functions are gapped around the Fermi energy in the entire $U$ range. At weak $U$ the system possesses charge order only; staggered magnetization is zero (cf. Fig. 3A ). By increasing $U m_{C D W}$ is reduced and AF correlations develop. The local interaction $U$ reduces the amount of double occupancy - needed to maintain a finite $m_{C D W}$ at half-filling. On the other hand, virtual hopping processes induce an effective exchange interaction, which favors antiferromagnetism. Beyond a critical $U(\Delta)$ the system acquires Néel order (cf. Fig. 3 B - E) accompanied by a strong reduction of the CDW amplitude.

In Fig. 4 we present how the order parameters $m_{\mathrm{CDW}}$ and $m_{\mathrm{AF}}$ vary with the interaction $U$ for different ionic potentials $\Delta$. As discussed above, due to the suppression of double occupancies by the on-site repulsion, the charge density wave order parameter is reduced but $m_{\mathrm{CDW}}>0$ for all $U$ as inferred from symmetry arguments. Whereas at $\Delta=0$ Néel order appears at infinitesimally small $U$, at finite $\Delta$ the interaction has to exceed a finite critical value $U_{c}(\Delta)$ for the onset of antiferromagnetism. This quantum phase transition at $U=U_{c}(\Delta)$ is continuous 


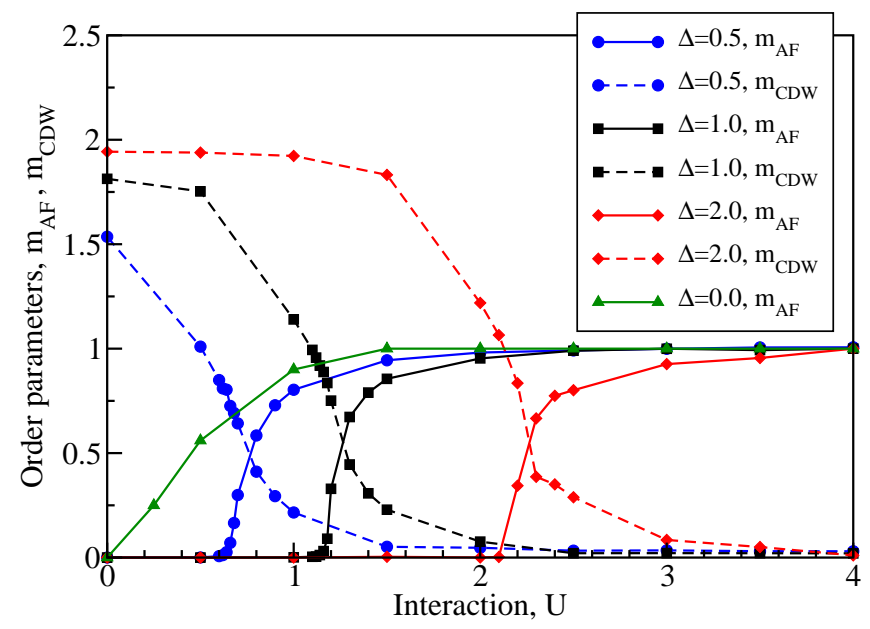

FIG. 4: (color online) $U$ dependence of spin- and chargedensity wave order parameters for different ionic potentials $\Delta$.

in contrast to the paramagnetic-antiferromagnetic transition for models with frustration. ${ }^{25}$ At very large $U$ the $\mathrm{AF}$ order parameter saturates at its maximum value.

In summary, our numerical solution of the DMFT equations for the ionic Hubbard model provides evidence for the existence of a critical interaction strength for the transition from a weakly correlated band insulator to a Mott insulator with coexisting charge and staggered spin order. A gap in the one-particle spectrum persists in all parameter regimes and thus implies the absence of an intervening metallic phase. We emphasize that our results do not contradict the findings in Refs. 11, 12,13, 14 because in those works the AF long-range order was either excluded by the choice of the method or by the low dimensionality of the system and finite temperatures. Transitions between a correlated metal and a band insulator are also found in other models, such as the Hubbard model with binary alloy disorder ${ }^{26.27}$ or the bilayer Hubbard model with interlayer hopping. ${ }^{28}$ As we have demonstrated here, the occurrence of spontaneous staggered long-range order can significantly change the nature or even the existence of a transition to a metallic phase.

We thank D. Vollhardt for insightful discussions. This work was supported by the Sonderforschungsbereich 484 of the Deutsche Forschungsgemeinschaft (DFG). The main part of the calculations were performed when K.B. was at Augsburg University.
1 F. Gebhard, The Mott Metal-Insulator Transition: Models and Methods (Springer, 1997).

2 J. Hubbard and J. B. Torrance, Phys. Rev. Lett. 47, 1750 (1981).

3 N. Nagaosa and J. Takimoto, J. Phys. Soc. Jpn. 55, 2735 (1986).

4 T. Egami, S. Ishihara, and M. Tachiki, Science 261, 1307 (1993); S. Ishihara, T. Egami, and M. Tachiki, Phys. Rev. B 49, 8944 (1994).

5 J. Kuneš and V. I. Anisimov, Phys. Rev. B 78, 033109 (2008).

6 S. Fölling et al., Nature 448, 1029 (2007); M. Greiner and S. Fölling, Nature 453, 736 (2008); S. Trotzky et al., Science 319, 295 (2008).

7 M. Fabrizio, A. O. Gogolin, and A. A. Nersesyan, Phys. Rev. Lett. 83, 624 (1999).

8 A. P. Kampf, M. Sekania, G. I. Japaridze, and P. Brune, J. Phys.: Condens. Matter 15, 5895 (2003).

9 S. R. Manmana, V. Meden, R. M. Noack, and K. Schönhammer, Phys. Rev. B 70, 155115 (2004).

10 T. Jabben, N. Grewe, and F. B. Anders, Eur. Phys. J. B 44, 47 (2005).

11 A. Garg, H. R. Krishnamurthy, and M. Randeria, Phys. Rev. Lett. 97, 046403 (2006).

12 L. Craco, P. Lombardo, R. Hayn, G. I. Japaridze, and E. Müller-Hartmann, Phys. Rev. B 78, 075121 (2008).

13 S. S. Kancharla and E. Dagotto, Phys. Rev. Lett. 98, 016402 (2007).

14 N. Paris, K. Bouadim, F. Hebert, G. G. Batrouni, and R. T. Scalettar, Phys. Rev. Lett. 98, 046403 (2007); K. Bouadim, N. Paris, F. Hebert, G. G. Batrouni, and R. T. Scalettar, Phys. Rev. B 76, 085112 (2007).
${ }^{15}$ For a review see: A. Gelfert, arXiv:cond-mat/0106031 (unpublished); A. Gelfert and W. Nolting, J. Phys.: Condens. Matter 13, R505 (2001).

16 W. Metzner and D. Vollhardt, Phys. Rev. Lett. 62, 324 (1989).

17 D. Vollhardt, Correlated Electron Systems, Vol. 9, Ed. V. J. Emery (World Scientific, Singapore, 1993), p. 57.

18 Th. Pruschke, M. Jarrell, and J. K. Freericks, Adv. Phys. 44, 187 (1995).

19 A. Georges, G. Kotliar, W. Krauth, and M. J. Rozenberg, Rev. Mod. Phys. 68, 13 (1996).

20 R. Bulla, Th. Costi, and Th. Pruschke, Rev. Mod. Phys. 80, 395 (2008) and references therein.

21 R. Bulla, Phys. Rev. Lett. 83, 136 (1999).

22 M. Eckstein, M. Kollar, K. Byczuk, and D. Vollhardt, Phys. Rev. B 71, 235119 (2005).

23 M. Kollar, M. Eckstein, K. Byczuk, N. Blümer, P. van Dongen, M. H. Radke de Cuba, W. Metzner, D. Tanaskovic, V. Dobrosavljevic, G. Kotliar, and D. Vollhardt, Ann. Phys. (Leipzig) 14, 642 (2005).

24 R. Bulla, T. A. Costi, and D. Vollhardt, Phys. Rev. B 64, 045103 (2001)

25 R. Zitzler, N. Tong, Th. Pruschke, and R. Bulla, Phys. Rev. Lett. 93, 016406 (2004).

${ }^{26}$ K. Byczuk, W. Hofstetter, and D. Vollhardt, Phys. Rev. B 69, 045112 (2004).

27 M. Balzer and M. Potthoff, Physica B 359-361, 768 (2005).

28 A. Fuhrmann, D. Heilmann, and H. Monien, Phys. Rev. B 73, 245118 (2006). 